\title{
Exercising Power in Software Ecosystems
}

\author{
Carina Alves ${ }^{1}$, George Valença ${ }^{2}$, Xavier Franch ${ }^{3}$ \\ ${ }^{1}$ Universidade Federal de Pernambuco, Centro de Informática, Recife, Brazil. \\ ${ }^{2}$ Universidade Federal Rural de Pernambuco, Departamento de Computação, \\ Pernambuco, Brazil. \\ ${ }^{3}$ Universitat Politècnica de Catalunya, Software and Service Engineering Group, \\ Barcelona, Spain.
}

\begin{abstract}
A key principle of software ecosystems is that players aim to achieve mutual benefits by contributing towards their growth. The transformation of software products into platforms requires a different business mindset. We observed that understanding the dynamics of power is fundamental for the effective management of software ecosystems. Companies in an ecosystem must understand which power capabilities can drive cooperation or generate conflicts. In this paper, we propose an approach for ecosystem players to exercise power. We also analyse how power influences the relationships among companies in ecosystems formed by Small-toMedium Enterprises as well as in platform ecosystems governed by large keystones.
\end{abstract}

Software ecosystems are bringing radical changes in the way software companies operate. The battleground is no longer between competing products. Instead, it involves ecosystems shaped around them. The transformation of products into platforms requires a different business mindset. Companies establish partnerships to integrate complementary solutions or extend the central technology offered by a platform owner.

To succeed in a software ecosystem, companies must identify core power capabilities to achieve their goals. For instance, a company that develops innovative apps can strengthen its position or alter its role in the ecosystem. As a result, a company can raise its status among partners, given their recognition of such capability. Another source of power that a company can explore is sharing business opportunities with partners in the ecosystem, which is a regular action taken by keystones such as Apple and SAP through their marketplaces. In these cases, companies aim the exercise of power brings value and contributes towards the growth of the ecosystem. In different contexts, companies may have to deal with the centrality and manipulation of a powerful player, face punitive actions in light of a high power imbalance, or hold a weak and vulnerable position due to lack of relevant resources. These situations show the importance of analysing the dynamics of power for effectively managing a software ecosystem.

In recent research [1,2], we conducted multiple case studies of emerging software ecosystems formed by Small-to-Medium Enterprises (SMEs). We proposed a theory of power to explain how power relationships are established between ecosystem partners [1]. Our practical contributions were twofold. We investigated the structure of power relationships between partners, based on a careful analysis of their power capabilities. Besides, we examined how power affects the behaviour and coordination of SMEs participating in an ecosystem [2]. All data of the case studies are available at https://sites.google.com/site/powerinsoftwareecosystems/. In the present article, we go 
one step beyond by proposing an approach for companies to identify and apply power. We also illustrate the dynamics of power in platform ecosystems structured by big players and in ecosystems raised by close relationships among SMEs.

\section{Understanding Power}

Social scientists have studied power for decades in interpersonal relationships. This concept was later introduced in business scenario by behavioural scientists and management professionals. We define the power of $A$ as the ability that $A$ has to exert some sort of influence in its relationship with $B$. This power generally stems from the dependence of $B$ upon $A$. It means that $A$ has fertile ground to exercise power over other player if the player somehow depends on $A$. We describe five types of power that a company can hold in a given relationship, considering the well-known taxonomy proposed by French and Raven [3]:

- Coercive Power is the perception of $B$ that $A$ has ability to punish it, e.g. a company disqualifies partners whose products do not live up to quality standards;

- Reward Power is the perception of $B$ that $A$ has ability to offer rewards, e.g. a company provides financial benefits to partners in the ecosystem;

- Expert Power is the perception of $B$ that $A$ has special knowledge or expertise, e.g. a company has strategic market knowledge or masters innovative technologies;

- Legitimate Power is the perception of $B$ that $A$ has the right to impose behaviour for it, e.g. a company can set ecosystem goals due to its superior position.

- Referent Power is the feeling of respect or admiration from B towards A, e.g. players value a company due to their recognition of its status, which creates a feeling of identification and attracts them.

We define power capability as a given asset that denotes the power of a company, such as developing functionalities for a specific market segment, providing partners with key information about customers, or defining the roles of partners in a joint initiative for systems integration. Each power capability derives from one or more power sources, which represent tangible or intangible resources that an actor can use to affect the behaviour of another [1]. Hence, by cultivating such sources, a company is able to exercise some form of power. In particular, any change in the availability or demand for power sources may affect power distribution in a partnership, since it causes an actor to obtain or lose power.

\section{Power in Software Ecosystems}

The player in charge of providing a central technology (e.g. from a datahub to a complete and vibrant software platform) and managing the relationships among players is known as keystone. This company controls the ecosystem by defining governance mechanisms, such as establishing entry requirements, stimulating partner investments and sharing knowledge [4]. The ability of the keystone to create a prosperous ecosystem where value is distributed among all participants is vital for this player to ensure a strategic position in the market. Sometimes, it is a condition for a company to thrive or die.

Ecosystems orchestrated by large companies have a clear and quite stable structure of power. On the one side, third-party developers, known as complementors, are attracted to 
the platform willing to obtain advantages, such as money and networking. On the other side, the keystone relies on apps and extensions built by complementors. Therefore, both sides mutually depend on each other. For instance, Microsoft Dynamics heavily depends on resellers and independent software vendors, such as the CRM specialists BusinessBase and Isatech, to expand the ecosystem. In their turn, these small partners obtain great opportunities for revenue growth by reaching the large pool of customers from Dynamics CRM through Microsoft marketplace [5]. The high dependence of complementors on superior financial and technological resources from the keystone provides it with some prerogatives over them, such as the authority to define rules that complementors should obey. This resource asymmetry causes an unbalanced power structure [6]. The keystone is notably the most powerful actor due to its size and capabilities. However, putting it simply, the keystone cannot survive alone without the inventive collaboration of partners.

Ecosystems can also result from recurrent partnerships among SMEs. For example, Veeqo (https://developer.veeqo.com) is a visionary small company providing ecommerce solutions that is fostering a developer ecosystem by creating a partner program on top of its platform, with an open API and expert support. Generally, SMEs join forces to integrate their software solutions as a strategy to increase their competitive advantage. Our case studies revealed that, in this type of software ecosystem, power is widely diffused among players [2]. One of the studied SME, here called Company A, has strong expertise in enterprise procurement solutions. This knowledge is a source of power for the company during negotiations to decide the vendor that will implement a strategic part of the integrated solution. In another software integration project, Company A integrates its solution with Company $\mathrm{B}$, which is a local market leader with a relevant pool of customers. In this case, Company B has the authority to impose certain conditions (e.g. negotiate pricing deals directly with clients, define technical specifications of the integration infrastructure) that Company A needs to accept. The relationship among these companies is grounded on rules that are more flexible, such as a rotating leadership arrangement, instead of fixed keystone and complementors roles. Hence, ecosystems formed by SMEs tend to keep more fluid power relationships.

From this analysis, we can highlight two main differences in the structure of large platform ecosystems and ecosystems formed by SMEs. The first one concerns the management of the ecosystem. In platform ecosystems, the keystone provides the platform and governs the network. In their turn, in ecosystems of SMEs, the management is distributed among participants who contribute to build a shared infrastructure. Commonly, the SME holding more sources of power in a particular situation gains the leadership position. The second difference involves the exercise of power. In platform ecosystems, the keystone continuously exercises greater power over complementors. In contrast, in ecosystems formed by SMEs, power tends to be more balanced and in constant change among participants, given their similar size and comparable levels of resources and capabilities.

\section{An Approach for Exercising Power}

We can condense the findings from our multiple case studies into a three-step approach for companies to exercise power in software ecosystems. Initially, a company identifies main power capabilities by considering key resources and distinctive abilities that may originate power over other players. For instance, a company has to analyse when and which (i) expertise is crucial to create value for a relationship and enable the ecosystem to flourish (Expert Power); (ii) right is available in a joint initiative (Legitimate Power); 
(iii) penalty can be applied over partners (Coercive Power); and so on. These power capabilities must be associated with a power type. Since power capabilities are key assets owned by a company, they act as success criteria to enter and prosper in a software ecosystem.

Secondly, a company structures power relationships with players through a critical analysis of power capabilities. A company must consider its motivation for cooperation, business strategies, current position, and potential areas of conflict. Moreover, since power is a relative notion, it is paramount that a company identifies the main power capabilities of key partners in the ecosystem, as well as understands the dependence kept on and generated over them. Then, the company may decide to apply power-balancing operations to promote structural changes in power relationships by altering the power advantage [6]. For instance, a complementor of Microsoft Dynamics ecosystem can exploit its knowledge of CRM domain (Expert Power capability) to develop innovative features that may provide the complementor with intellectual property rights (power source). The complementor can use this resource to raise the dependence of Microsoft or other partners, reinforce its Expert Power in the ecosystem and even generate a new power capability.

A company must also understand in which order their power capabilities shall be used. For instance, keystones such as Apple aim to enable a whole ecosystem of complementors to thrive around its platform. Establishing a diverse marketplace of mobile apps in the iOS ecosystem is a means for Apple to outperform competitors. Keystones need to nurture capabilities that are beneficial for the network and desired by other players. Therefore, keystones can use the appeal of their Referent Power to attract partners to the network. In parallel, they must clearly exercise Reward Power to guarantee a win-win approach in the relationships. In particular, this is a tactic to lock-in players by raising their dependence on the benefits obtained in the ecosystem. Then, it is important to use Legitimate Power to establish governance rules that will guide the cooperation among participants. For instance, in the "PartnerEdge Program for Application Development", SAP stresses its status of leading enterprise software vendor. The company guarantees the access of partners to a development platform with multiple cutting-edge technologies, on top of which they can build applications and release them to a large customer base in its marketplace. Besides, SAP increases the benefits shared with a partner company according to its contribution to the ecosystem. This rule motivates partners while regulating their dependence on the program, which entitles SAP to exercise power over them (https://www.sap.com/partner/become.programoptions.html).

Finally, a company monitors the results of power exercise. Through performance indicators, it can assess how the established power relationships have supported its goals as well as that of partners and the ecosystem as whole. If the exercise of power is not effective, a company must take actions to create, reinforce or avoid specific power capabilities, based on the sources of power available in the ecosystem. During this step, a company may also perceive changes of power over time, since each power type has a tendency to transform into or originate another form of power [7]. As an example, one company may perceive that its Reward Power of certifying partners and granting benefits based on their roles has reinforced its Referent Power or promoted the Legitimate Power to occupy a leadership position in the ecosystem.

\section{Using Power Wisely}

In our studies, we observed that the most important and frequent power types used by 
players is Reward Power [2]. Involving partners in system integration projects or providing them with strategic resources are main drivers to establish new relationships or consolidate current ones. For instance, in one case study, two SMEs, here named here as Company A and Company C, shared critical information about the market (e.g. potential feature requests from big customers, market prospects, etc.) with Company B. In a different way, the exercise of Coercive Power involves punishment and hostile behaviour. This power type is not commonly employed for several reasons. Only companies that keep a short dependence on partners, control key resources, or hold a strategic position can use Coercive Power. For instance, Microsoft may exercise Coercive Power by applying sanctions over complementors to impose its choices. The company used to threaten to halt the development of Microsoft Office for Mac computers in case Apple no longer adopted Microsoft's web browser [10]. However, the frequent exercise of Coercive Power may encourage players to leave the ecosystem. It means that coercion brings the risk that one company may obtain most or all of the value within the ecosystem at the expense of others. Therefore, this situation of dominance is not healthy for the relationships in an ecosystem.

Coercive and Reward Power are examples of what is called hard power [8], because they involve a significant degree of dependence of companies on the power holder. These types of power are quite advantageous for dominant companies, which can easily control players by imposing restrictions or granting incentives for partners. Hence, there is an underlying notion of threat in exercising such power types, such as the possibility of being punished by another party or losing an important benefit. A common side effect of constantly using hard power is that it affects the trust among players. Trusting other players is vital to build prosperous relationships [9]. To promote collaboration, companies must trust other players will not take advantage of their vulnerabilities. Companies that do not have autonomy or capabilities to exert some type of power must accept their weaknesses and make a strong leap of faith on partners.

Instead of relying on measures of payment or punishment, companies should also explore their knowledge, rights, and respect to apply Expert Power, Legitimate Power and Referent Power over players. These are examples of what can be called soft power [8]. SMEs normally use soft power because they do not have the money or authority of a large company. Weaker players can obtain a relevant role or facilitate negotiations in the ecosystem by fostering capabilities that enable them to use these types of power. Expert Power, Legitimate Power and Referent Power require sources of power attainable by SMEs, such as the ability to develop innovative product features, responsibility for a new business deal, and good reputation with customers, respectively.

\section{Consequences of Power}

Companies must exercise power in a wise manner. For instance, a company may decide to exploit its power capabilities in order to adopt a dominator role as strategy. In this case, a company may use Reward Power to grant partners with benefits; but, at the same time, it defines limited rights for partners that will place them in a weaker and uncomfortable situation. The company is extracting maximum value from other players and absorbing the network [10]. This example remarks that power influences individual relationships between parties, but the overall consequences of power exercise may affect the entire ecosystem. Therefore, based on our previous studies, we recommend companies to build suitable power-dependence relationships to ensure their own success as well as the success of the ecosystem. It is a delicate equilibrium of conflicting forces. In the end, companies may be able to manage the subtle tensions of power and 
dependence in their relationships by following Sun Tzu's ancient advice "appear weak when you are strong, and strong when you are weak" [11].

\section{References}

1. Valença, G. Alves, C. A Theory of Power in Emerging Software Ecosystems Formed by Small-to-Medium Enterprises. The Journal of Systems and Software. pp. 76-104, n 134, 2017.

2. Valença, G. Alves, C. The Dynamics of Power in Software Ecosystems: Insights from a Multiple Case Study. $43^{\text {rd }}$ Euromicro Conference on Software Engineering and Advanced Applications (SEAA), pp. 66-73, 2017.

3. French, J. Raven, B. The bases of social power. Cartwright D (ed). Studies in Social Power, pp. 150-167, 1959.

4. van Angeren, J. Alves, C. Jansen, S. Can We Ask You To Collaborate? Analyzing App Developer Relationships in Commercial Platform Ecosystems. Journal of Systems and Software, Volume 113, pp. 430-445, 2016.

5. https://msdynamicsworld.com/story/13-companies-microsoft-dynamics-ecosystem-made2015-inc-5000

6. Emerson, R. Power-dependence Relations. American Sociological Review, pp. 31-41, 1962.

7. Wrong, D. H., 1980. Power: Its forms, bases, and uses. Transaction publishers.

8. Nye, J. S.Soft Power: The Means To Success In World Politics. PublicAffairs, 2009.

9. Hurni, T. Huber, T. The Interplay of Power and Trust in Platform Ecosystems of the Enterprise Application Software Industry. In European Conference on Information Systems, pp. 1-15, 2014.

10. Yoffie, D. Kwak, M. With friends like these: the art of managing complementors. Harvard Business Review, September issue, 2006.

11. Tzu, S. The Art of War. Chartwell Books. 2012. 


\begin{abstract}
A key principle of software ecosystems is that players aim to achieve mutual benefits by contributing towards their growth. The transformation of software products into platforms requires a different business mindset. We observed that understanding the dynamics of power is fundamental for the effective management of software ecosystems. Companies in an ecosystem must understand which power capabilities can drive cooperation or generate conflicts. In this paper, we propose an approach for ecosystem players to exercise power. We also analyse how power influences the relationships among companies in ecosystems formed by Small-to-Medium Enterprises as well as in platform ecosystems governed by large keystones.
\end{abstract}

\title{
Keywords
}

D.2 Software Engineering < D Software/Software Engineering, D.2.9 Management < D.2 Software Engineering < D Software/Software Engineering, D.2.9.e Organizational management and coordination < D.2.9 Management < D.2 Software Engineering < D Software/Software Engineering, K.6.3 Software Management < K.6 Management of Computing and Information Systems < K Computing Milieux, M.9.0.a Dynamics of Services Ecosystem < M.9.0 General < M.9 Enterprise Modeling and Management $<$ M Services Computing, M.6.2.ia Inter-Enterprise Collaboration < M.6.2 Services Value Chain Collaboration < M.6 Services Composition < M Services Comput, K.6.1.d Strategic information systems planning < K.6.1 Project and People Management < K.6 Management of Computing and Information.

\section{References}

1. Valença, G. Alves, C. A Theory of Power in Emerging Software Ecosystems Formed by Small-to-Medium Enterprises. The Journal of Systems and Software. pp. 76-104, n 134, 2017.

2. Valença, G. Alves, C. The Dynamics of Power in Software Ecosystems: Insights from a Multiple Case Study. 43rd Euromicro Conference on Software Engineering and Advanced Applications (SEAA), pp. 66-73, 2017.

3. French, J. Raven, B. The bases of social power. Cartwright D (ed). Studies in Social Power, pp. 150-167, 1959.

4. van Angeren, J. Alves, C. Jansen, S. Can We Ask You To Collaborate? Analyzing App Developer Relationships in Commercial Platform Ecosystems. Journal of Systems and Software, Volume 113, pp. 430-445, 2016.

5. https://msdynamicsworld.com/story/13-companies-microsoft-dynamics-ecosystem-made2015-inc-5000

6. Emerson, R. Power-dependence Relations. American Sociological Review, pp. 31-41, 1962.

7. Wrong, D. H., 1980. Power: Its forms, bases, and uses. Transaction publishers.

8. Nye, J. S.Soft Power: The Means To Success In World Politics. PublicAffairs, 2009.

9. Hurni, T. Huber, T. The Interplay of Power and Trust in Platform Ecosystems of the Enterprise Application Software Industry. In European Conference on Information Systems, pp. 1-15, 2014. 
10. Yoffie, D. Kwak, M. With friends like these: the art of managing complementors. Harvard Business Review, September issue, 2006.

11. Tzu, S. The Art of War. Chartwell Books. 2012.

\section{Short author bios}

CARINA ALVES is an associate professor at Universidade Federal de Pernambuco. Her research focuses on software ecosystems, requirements engineering and business process management. Alves received her $\mathrm{PhD}$ in Computer Science from University College London. Contact her at cfa@cin.ufpe.br.

GEORGE VALENÇA is an adjunct professor of software engineering at Universidade Federal Rural de Pernambuco. His research interests include software ecosystems, evidence-based and experimental software engineering, and business process management. He received a PhD in computer science from Universidade Federal de Pernambuco. Contact him at george.valenca@ufrpe.br.

XAVIER FRANCH is a professor of Informatics at Universitat Politècnica de Catalunya. His main research interests are requirements engineering, software evolution, software quality and software ecosystems. Contact him at franch@essi.upc.edu.

\section{Email address of each author}

cfa@cin.ufpe.br

george.valenca@ufrpe.br

franch@essi.upc.edu

Color photograph of each author (at least 245 pixels width and 300 pixels height) 

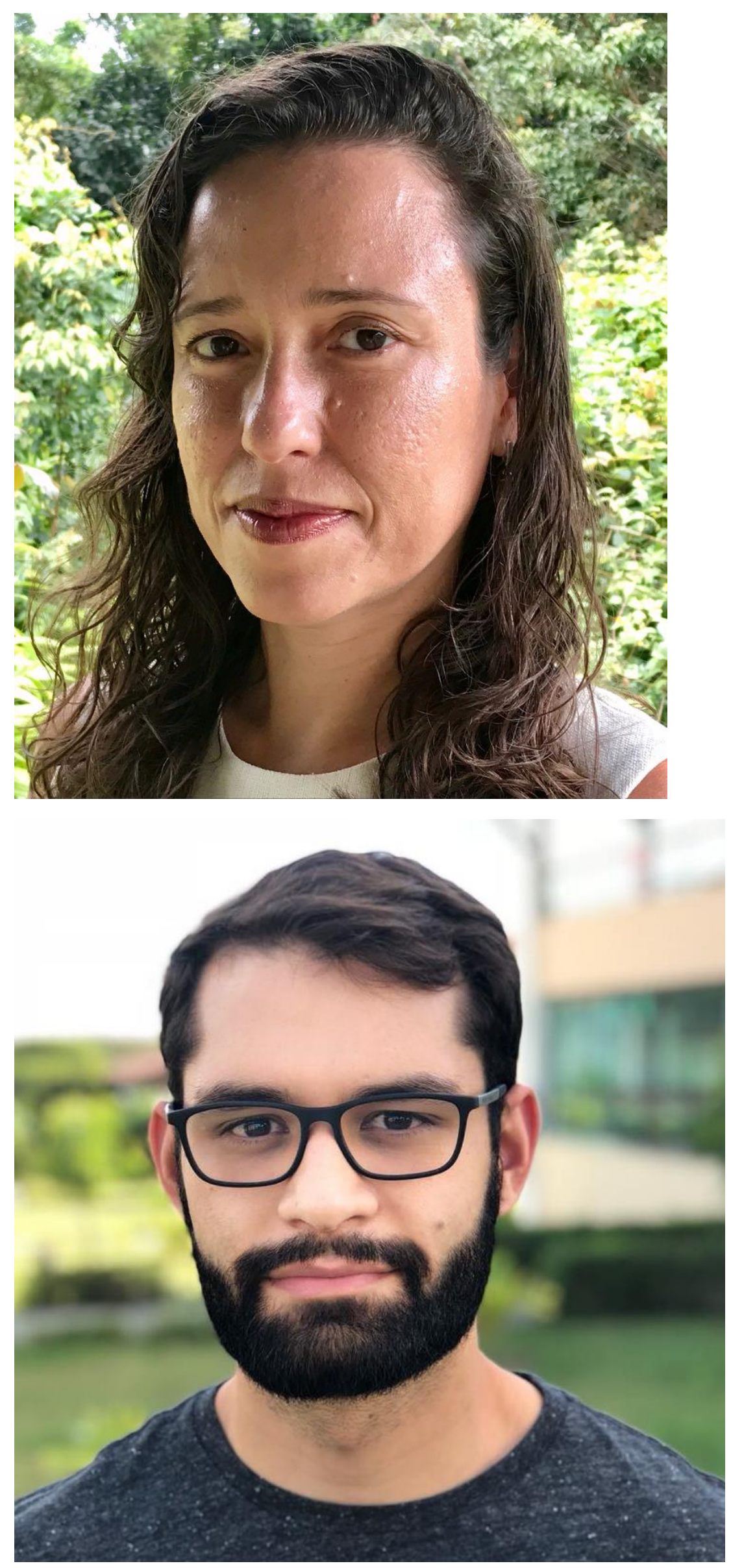


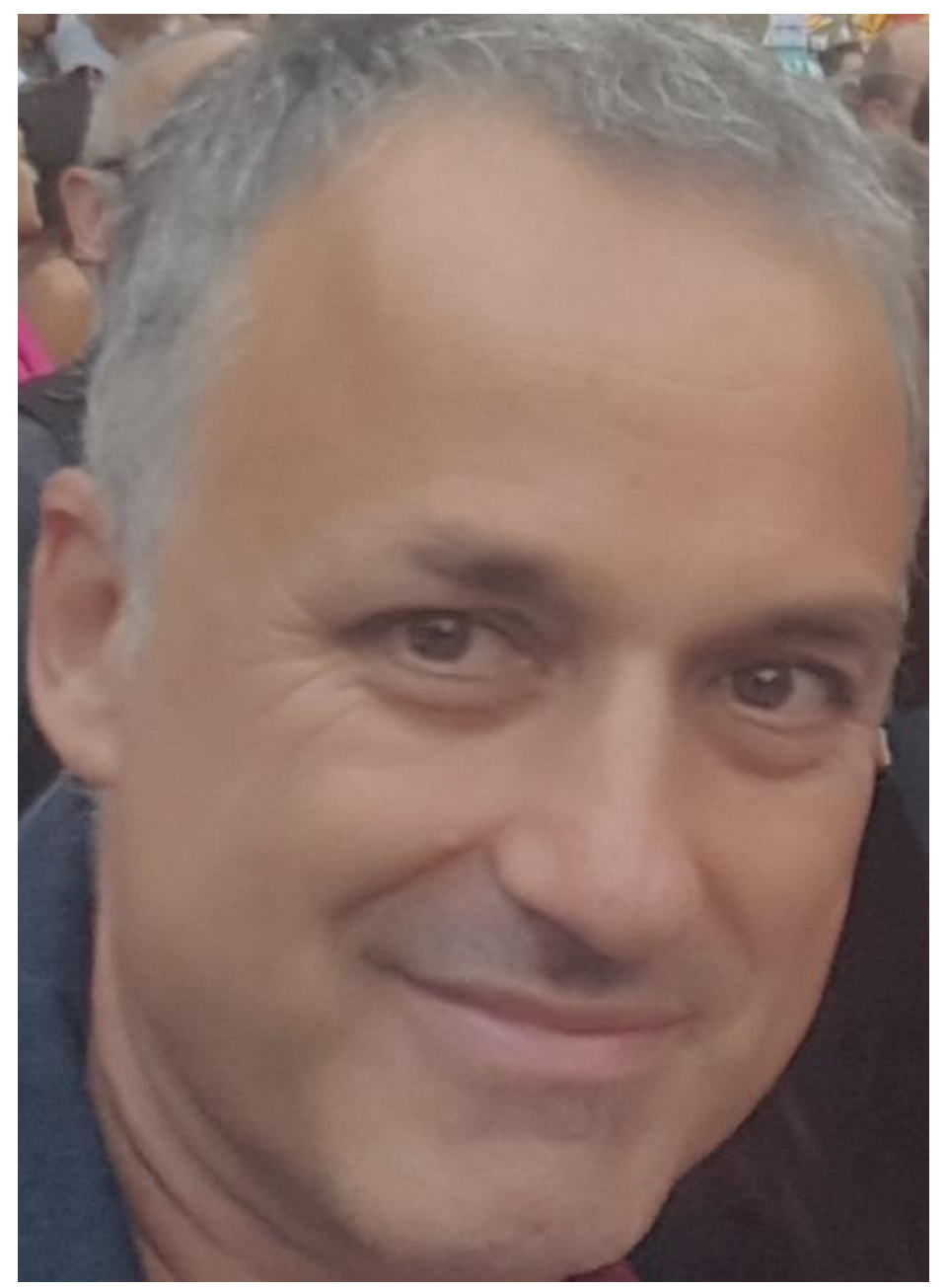

Three to five "tweets" (messages of 115 characters or less) each summarizing an interesting question or finding associated with your article.

Exercising power in a wise manner is fundamental for the effective management of software ecosystems.

Keystones should leverage their reward power to nurture ecosystem community.

Keystones should highlight their referent power to gain a needed authority over complementors.

Complementors should reinforce their expert power to bring value to the ecosystem and then gain legitimate power. 